\title{
YAP Deficiency Attenuates Pulmonary Injury Following Mechanical Ventilation Through the Regulation of MI/M2 Macrophage Polarization
}

This article was published in the following Dove Press journal: Journal of Inflammation Research

\section{Qiong Luo \\ Jing Luo \\ Yanlin Wang (iD}

Department of Anesthesiology,

Zhongnan Hospital of Wuhan University, Wuhan, Hubei 43007I, People's Republic of China
Correspondence: Yanlin Wang Department of Anesthesiology, Zhongnan Hospital of Wuhan University, Wuhan, Hubei 43007I, People's Republic of China Email ylwang917@163.com
Background: Evidences indicate that the balance between macrophage M1 and M2 polarization is essential for the regulation of pulmonary inflammation during mechanical ventilation (MV). Yes-associated protein (YAP) is a key component of the Hippo pathway and was suggested to regulate macrophage polarization. This study was designed to investigate whether YAP contributes to pulmonary inflammation during MV.

Methods: Wild-type and macrophage YAP knockout mice were mechanically ventilated for 12 hours to induce pulmonary injuries. At the end of MV, animals were sacrificed for pulmonary tissue collection and macrophage isolation. In addition, the induction of macrophage polarization was performed in isolated macrophages with or without YAP overexpression in vitro. Pulmonary injuries, YAP expression, macrophage polarization and cytokines were measured.

Results: Here, we show that MV induces lung injury together with pulmonary inflammation as well as upregulated YAP expressions in pulmonary macrophages. In addition, our results indicate that YAP deficiency in macrophages attenuates pulmonary injury, accompanied with decreased production of pro-inflammatory cytokines including IL (interleukin)-1 $\beta$, IL-6 and tumor necrosis factor-alpha (TNF- $\alpha)$. Moreover, both in vivo and in vitro studies indicate that YAP deficiency enhances M2 polarization while inhibits M1 polarization. In contrast, YAP overexpression inhibits the induction of M2 polarization but improves M1 polarization.

Conclusion: Our results report for the first time that the induction of YAP in macrophages contributes to pulmonary inflammation during MV through the regulation of M1/M2 polarization.

Keywords: yes-associated protein, macrophage polarization, pulmonary inflammation, mechanical ventilation

\section{Introduction}

Mechanical ventilation (MV) is one of the most commonly used life-support techniques in clinical practice. ${ }^{1}$ However, prolonged MV may worsen pulmonary inflammation, which could induce additional morbidity/mortality among critically ill patients. $^{2,3}$ Previous findings indicated that macrophage polarization plays a crucial role in the regulation of pulmonary inflammation following $M V^{4,5}$ Macrophages can polarize into two functional phenotypes in different microenvironments: classically activated inflammatory (M1) or alternatively (M2) activated macrophages. $^{6}$ In response to stimulus, M2 macrophages can express antiinflammatory cytokines while M1 macrophages secrete pro-inflammatory 
cytokines. $^{7}$ Then, the imbalance between M1/M2 macrophage polarization may result in excessive production of pro-inflammatory cytokines and serves as an important mechanism of inflammatory response. ${ }^{8}$ To date, the underlying mechanism by which MV induces the imbalance of M1/M2 macrophage polarization in pulmonary tissues has not yet been completely understood.

Yes-associated protein (YAP) is a key mediator of the Hippo signaling pathway, and plays important roles in promoting cancer development and drug resistance. ${ }^{9}$ Recently, it has been demonstrated that YAP participates in the development of inflammatory disease; however, the results are controversial. Yang and colleagues reported that YAP suppresses vascular inflammation through preventing the activation of NF- $\mathrm{KB}$ signaling activation. ${ }^{10}$ In contrast, Zhou and others demonstrated that YAP worsens inflammatory bowel disease by regulating M1/M2 macrophage polarization. ${ }^{11}$ All in all, those results demonstrated a tight association between YAP expression/activation and the pathogenesis of inflammation. The connective tissue growth factor (CTGF), a downstream gene of YAP, has been reported to be involved in inflammatory diseases ${ }^{12}$ and upregulated in acute lung injury (ALI). ${ }^{13} \mathrm{Xie}$ and colleagues demonstrated a significant correlation between mechanical power and CTGF in ARDS patients, and its level was related to the survival outcome. ${ }^{14}$ This study has particular significance to the early prevention of pulmonary injury in patients receiving MV.

However, it is unclear whether YAP is involved in MVinduced imbalance between pulmonary M1 and M2 macrophage polarization and subsequent inflammation. In the present study, we aim to determine the roles of YAP in the development of pulmonary inflammation following $\mathrm{MV}$ and the underlying molecular mechanism.

\section{Materials and Methods}

\section{Animals}

As previously reported, ${ }^{11}$ YAP fl/fl mice bearing two loxP sites flanking the first two exons of the YAP gene were cross-bred with Lysm-Cre mice to specifically knock out YAP in macrophages (termed $\mathrm{YAP}^{\triangle \mathrm{M} / \Delta \mathrm{M}}$ mice). In this study, we also used $\mathrm{YAP}^{\mathrm{f} / \mathrm{fl}} \mathrm{Lysm}^{\text {cre/wt }}$ as $\mathrm{YAP}^{\Delta \mathrm{M} / \Delta \mathrm{M}}$ and $\mathrm{YAP}^{\mathrm{f} / \mathrm{fl}} \mathrm{Lysm}^{\mathrm{wt} / \mathrm{wt}}$ as the control mice (termed $\mathrm{YAP}^{+/+}$). $\mathrm{YAP}^{+/+}$and $\mathrm{YAP}^{\Delta \mathrm{M} / \Delta \mathrm{M}}$ mice are in a $\mathrm{C} 57 \mathrm{BL} / 6$ background, which were purchased from Cyagen (Suzhou, China). Animals were kept in individual cages under standard conditions. All animal experiments were approved by the Animal Biosafety Level 3 Laboratory of Wuhan University (No. ZN2019011) and were performed in accordance with the Laboratory Guidelines for Animal Use and Care. ${ }^{15}$

\section{Mechanical Ventilation Model}

Mice were anesthetized with an intraperitoneal injection of sodium pentobarbital $(50 \mathrm{mg} / \mathrm{kg}$ ) (China Pharmaceutical Group, Shanghai, China). After 15 minutes of stabilization, animals were then tracheostomized and connected to a small animal ventilator (Harvard Apparatus, MA, USA) Ventilator-induced lung injury was achieved with the following parameters: ventilation time: 12 hours, respiration rate: 65 breaths $/ \mathrm{min}$, tidal volume: $20 \mathrm{~mL} / \mathrm{kg}$, and positive end-expiratory pressure (PEEP): $0 \mathrm{~cm} \mathrm{H}_{2} \mathrm{O}$. The bronchoalveolar lavage fluid (BALF) was obtained from three bronchoalveolar lavages in the upper part of the trachea by using $3 \mathrm{~mL}$ of PBS. Mice were sacrificed by blood dropping at the end of experiment.

\section{Isolation of Macrophages}

At the end of experiment, mice were sacrificed and the lungs were perfused with cold PBS and collected. Then, leukocytes were isolated from lungs by enzymatic digestion as previously described. ${ }^{16}$ After 90 -min adherence selection of digested tissue samples, non-adherent cells were rinsed away and the macrophages were collected in Trizol reagent. In addition, bone-marrow-derived macrophages (BMM) were isolated in accordance with the protocol as previously described. ${ }^{17}$ Briefly, the bone marrow suspension was centrifuged at $650 \times \mathrm{g}$ for $5 \mathrm{~min}$ at $4{ }^{\circ} \mathrm{C}$. Then, the cell pellet was re-suspended in $5 \mathrm{~mL}$ of lysis buffer $(8.3 \% \mathrm{NH} 4 \mathrm{Cl}, 0.1 \mathrm{M}$ Tris $)$ for $5 \mathrm{~min}$ to lyse the red blood cells. Again, the cell suspension was centrifuged at $650 \times \mathrm{g}$ for $5 \mathrm{~min}$ at $4{ }^{\circ} \mathrm{C}$. The cell pellet was collected and re-suspended in $5 \mathrm{~mL}$ of RPMI 1640 medium supplemented $10 \mathrm{ng} / \mathrm{mL}$ recombinant macrophage colony-stimulating factor (M-CSF). Cells were then incubated at $37^{\circ} \mathrm{C}$ and $5 \%$ $\mathrm{CO}_{2}$ for 1 week to ensure the bone marrow cells are fully differentiated into BMM. Then, BMM were removed from differentiation dishes using cold sterile PBS and cultured in RPMI 1640. Cells were washed with buffer (PBS with $0.5 \%$ bovine serum albumin and $0.02 \%$ sodium azide) for three times and stained with acid phosphatase. Cells were counted to ensure that the proportion of positively stained cells used in further experiments is higher than $90 \%$. 


\section{Flow Cytometric Analysis}

Isolated pulmonary macrophage cells were re-suspended in MACS buffer (BD Biosciences, CA, USA) and incubated with PE-conjugated anti-mouse CD11c (ab254183, Abcam), PE-conjugated anti-mouse F4/80 (ab6640, Abcam), and Alexa Fluor 647-conjugated CD206 (MCA2235A647, AbD Serotec), for $30 \mathrm{~min}$ at $4^{\circ} \mathrm{C}$. Cells were then washed and incubated with secondary antibodies for $30 \mathrm{~min}$ and then re-suspended in FACS buffer (BD Biosciences). Analysis was performed by a BD FACSCalibur flow cytometer (BD Biosciences) with FlowJo software (Tree Star, OR, USA). M1 and M2 macrophages were identified as $\mathrm{F} 4 / 80^{+} / \mathrm{CD} 11 \mathrm{c}^{+} / \mathrm{CD} 206$ and $\mathrm{F} 4 / 80^{+} / \mathrm{CD} 11 \mathrm{c}-/ \mathrm{CD} 206^{+}$, respectively.

\section{Induction of MI/M2 Polarization}

Culture medium on BMM was replaced with fresh medium, containing $25 \mathrm{mg} / \mathrm{mL}$ of IL4 and IL-13 or LPS (15 $\mathrm{ng} / \mathrm{mL})$ and IFN- $\gamma(50 \mathrm{ng} / \mathrm{mL})$ for M2 and M1 macrophage polarization, respectively. Cells were then cultured for 24 hours. At the time of harvest, cells were collected for the detection of M1/M2-related gene expressions by RT-PCR.

\section{Plasmid Preparation and Transfection}

The RAW264.7 cells were obtained from the American Type Culture Collection (ATCC) and cultured in DMEM (Life Technologies) containing 10\% (v/v) FBS. The overexpression of YAP was performed with the RAW264.7 cells. Cells were seeded in six-well plates and $2 \mu \mathrm{g}$ of YAP plasmid or pcDNA (vector) per well was transfected according to the manufacturer's instruction (Targeting Systems, CA, USA). The day before transfection, cells were plated on a 24 -well plate, $10^{5}$ cells per well. Cells were either transfected with YAP plasmid or vector. After $48 \mathrm{~h}$, cells were harvested and washed with fresh PBS for further analysis.

\section{Quantitative Real-Time PCR}

Total RNA was prepared using a Qiagen RNeasy fibrous mini-kit, using the protocol supplied by the manufacturer, and cDNA was produced using Prime Script RT-PCR Systems (Takara, Otsu, Japan). Quantitative real-time PCR was performed on a CFX-96 real-time PCR detection system (Bio-Rad). The primer sequences used in this study are listed as follows: IL-1 $\beta$ : forward primer: CTGGTACATCAGC ACCTCAC, reverse primer: AGAAACAGTCCAGCCCAT
AC; IL-6: forward primer: TGTATGAACAACGATG ATGCACTT, reverse primer: ACTCTGGCTTTGTCTTT CTTGTTATCT; IL-10: forward primer: CAGGGATCTTAG CTAACGGAAA, reverse primer: GCTCAGTGAATAA ATAGAATGGGAAC; Fizz1: forward primer: CCAATCC AGCTAACTATCCCTCC, reverse primer: ACCCAGTAGC AGTCATCCCA; Arg1: forward primer: CTCCAAGCCAA AGTCCTTAGAG, reverse primer: GGAGCTGTCATT AGGGACATCA; Ym1: forward primer: CAGGTCTGGCA ATTCTTCTGAA, reverse primer: GTCTTGCTCATGTG TGTAAGTGA; TNF- $\alpha$ : forward primer: AGTGACAAGC CTGTAGCCC, reverse primer: GAGGTTGACTTTCTCC TGGTAT; iNOS: forward primer: GGAGTGACGGCA AACATGACT, reverse primer: TCGATGCACAACTGG GTGAAC; CTGF: forward primer: GTGGAATATTGCC GGTGCA, reverse primer: CCATTGAAGCATCTTGGT TCG.

\section{Western Blot Analyses}

Western blotting was performed in a standard procedure. Tissue or cell lysates were prepared in RIPA buffer containing complete protease inhibitor cocktail and inhibitor for phosphatase. Protein concentration was determined using a BCA protein assay kit (Abcam, Shanghai, China). Proteins were then separated by a SDS-PAGE system. Membranes were incubated with primary antibodies including anti-YAP (Cell Signaling, MA, USA)), anti-CTGF (Abcam, Shanghai, China), anti-Arg1 (Abcam, Shanghai, China) and anti-iNOS (Abcam, Shanghai, China) at $4^{\circ} \mathrm{C}$ overnight and were probed with HRP-labeled secondary antibody. The membranes were visualized using an enhanced chemiluminescence system (Kodak, Rochester, USA). $\beta$-actin was used as a loading control.

\section{Cytokine Array Analyses}

The expression profile of cytokines was analyzed with commercial cytokine detection kits (R\&D Systems, Minneapolis, MN). Blocking, hybridization, washing conditions, and detection steps were performed according to the manufacturer's guidelines.

\section{Histologic Analyses}

Pulmonary tissues were fixed with $4 \%$ paraformaldehyde and embedded in paraffin. Then, sections were stained with hematoxylin and eosin (H\&E) by standard procedure. Pulmonary injuries were scored from 0 to 4 based on the degrees of edema, hemorrhage, infiltration of inflammatory cells and the histological changes as previously 
described. ${ }^{18} 0$ : no injury; 1: modest injury; 2: intermediate injury; 3: widespread injury; and 4: severe injury. These analyses were performed by two investigators in a blinded manner.

\section{Wet/Dry Ratio Assessment}

For each animal, the middle lobe of the left lung was removed at the end of experiment for wet/dry ratio assessment. In detail, pulmonary tissue was first weighted to determine the wet weight (ww). Then, tissues were dried at $80^{\circ} \mathrm{C}$ for 2 days to obtain the dry weight (dw). Finally, the wet/dry ratio was calculated as $(\mathrm{ww}-\mathrm{dw}) / \mathrm{dw}$.

\section{Immunofluorescence Staining}

Cells were fixed with $3.4 \%$ paraformaldehyde (PFA) (Maokang, Shanghai, China) for $20 \mathrm{~min}$ and then permeabilized with $0.5 \%$ Triton X-100 (Sigma-Aldrich). After blocking in 3\% PBS-BSA for $30 \mathrm{~min}$, slides were incubated with anti-YAP (1:200, Abcam, CA, USA). After washing with PBS, slides were incubated with goat anti-mouse FITC conjugated secondary antibodies for $1 \mathrm{~h}$ at $37^{\circ} \mathrm{C}$. The nuclei were stained using DAPI. The targeted proteins were detected using confocal microscopy (ZEISS LSM700) and a laserscanning confocal microscope image system. In addition, the immunofluorescence staining of pulmonary tissues was performed as previously described. ${ }^{19}$

\section{Statistical Analyses}

Data are presented as the mean \pm standard deviation (SD). Comparisons between two groups were made using the $t$-test. Differences among more than two groups were analyzed using one-way ANOVA followed by a Bonferroni post hoc test. A two-tailed $\mathrm{P}$ value less than 0.05 was considered significantly different.

\section{Results}

Mechanical Ventilation Induces Pulmonary Injury Together with Inflammation as Well as Upregulated YAP Expression

In the present study, 12 hours of mechanical ventilation with a very high tidal volume $(20 \mathrm{~mL} / \mathrm{kg})$ induced apparent pulmonary injuries as reflected by edema, hemorrhage, and infiltration of inflammatory cells (Figure 1A). In addition, the histological scores and pulmonary wet/dry ratio in the MV group were significantly increased as compared with that of the Control group $(p<0.001$, respectively) (Figure $1 \mathrm{~B}$ and $\mathrm{C}$ ). In order to evaluate the inflammatory response in pulmonary tissues after $\mathrm{MV}$, the expression of pro-inflammatory cytokines including TNF$\alpha$, IL- $1 \beta$ and IL-6 were measured. As seen in Figure 1DF, excessive expressions of TNF- $\alpha$, IL- $1 \beta$ and IL- 6 in injured lungs were documented after 12 hours of MV. Moreover, RT-PCR and western-blot assays suggested that YAP mRNA and protein expressions in injured lungs were significantly upregulated following prolonged MV (Figure $1 \mathrm{G}$ and $\mathrm{H}$ ). The connective tissue growth factor (CTGF), a downstream gene of YAP, was also significantly increased at both mRNA and protein levels in pulmonary tissues of animals after $\mathrm{MV}$ as compared with that of controls ( $p<0.05$, respectively). Together, these results indicated that prolonged $\mathrm{MV}$ induces pulmonary injury with excessive production of proinflammatory cytokines as well as upregulated YAP expression.

\section{YAP Deficiency in Pulmonary Macrophages Protects Mice Against Ventilator-Induced Lung Injury}

Considering the significant role of macrophage in pulmonary inflammation following $\mathrm{MV}$, we explored the function of YAP in macrophages with respect to pulmonary inflammation. As seen in Figure 2A, YAP deficiency in macrophages $\left(\mathrm{YAP}^{\triangle \mathrm{M} / \Delta \mathrm{M}}\right)$ resulted in reduced edema, hemorrhage and inflammatory cell infiltration. In addition, the histological scores in the $\mathrm{YAP}^{\Delta \mathrm{M} / \Delta \mathrm{M}}$ mice were significantly lower than that of wild-type mice $(p<0.001)$. These results indicate that YAP deficiency in macrophages protects mice against pulmonary injury following MV. The knockout efficiency of YAP in macrophages were determined by immunofluorescence staining, RT-PCR and western-blots (Figure 2B-E). In addition, ELISA assays showed that the production of pro-inflammatory cytokines including TNF- $\alpha$, IL-1 $\beta$ and IL-6 was significantly lower in $\mathrm{YAP}^{\Delta \mathrm{M} / \Delta \mathrm{M}}$ mice than that of wild-type mice $\left(\mathrm{YAP}^{+/+}\right)$ ( $p<0.001$, respectively) (Figure 2F-H). Collectively, these results suggested that YAP deficiency in pulmonary macrophages protects mice against ventilator-induced lung injury with downregulated production of pro-inflammatory cytokines.

\section{YAP Deficiency Enhances M2 Macrophage Polarization in vivo and in vitro}

Since M1/M2 polarization plays a crucial role in the pathogenesis of pulmonary inflammation during $\mathrm{MV}$, 

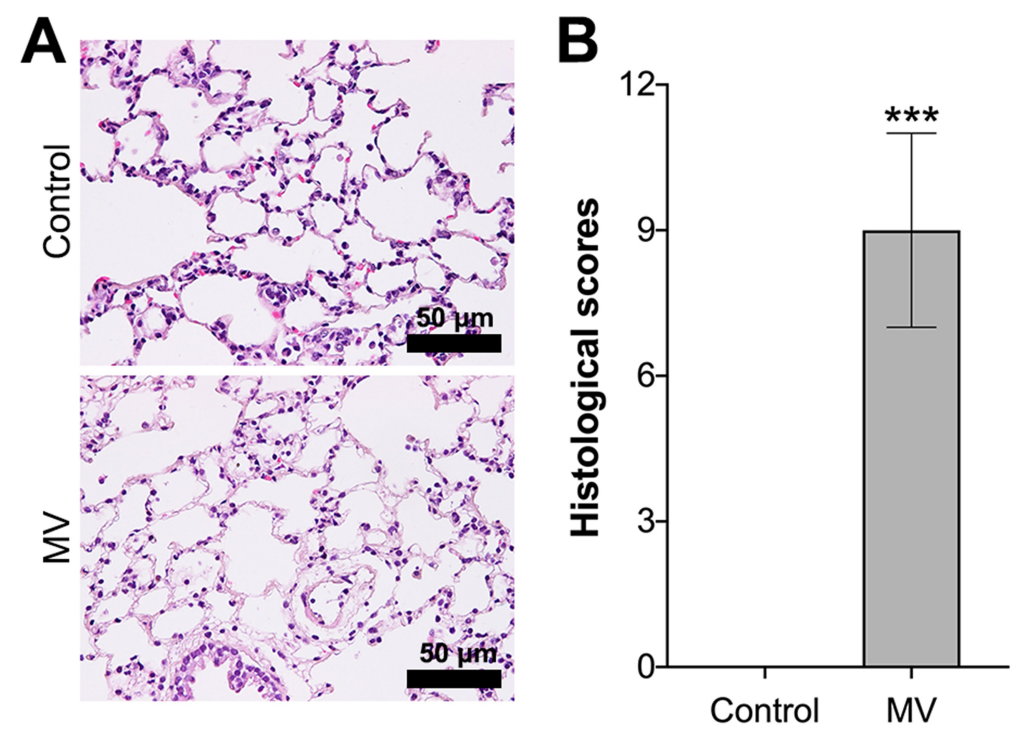

C
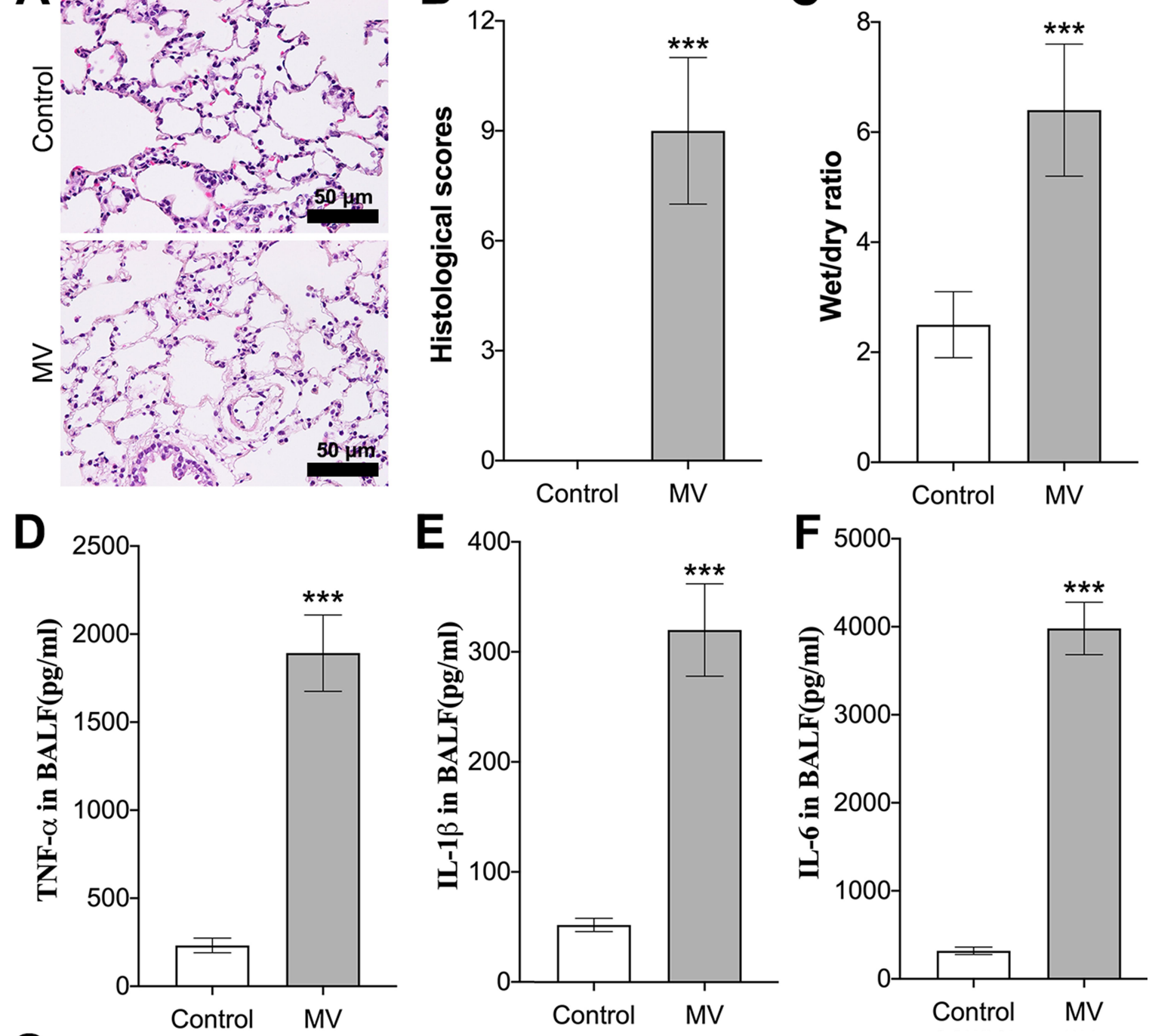

G
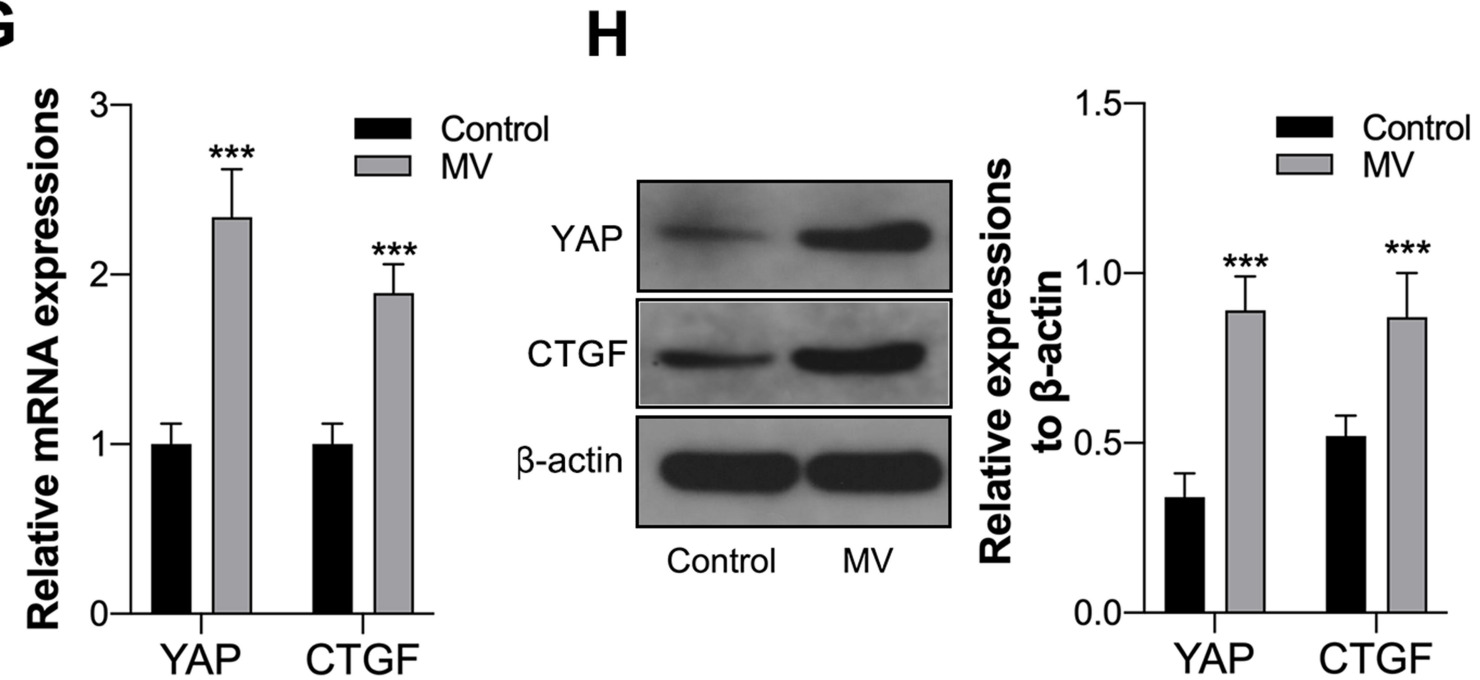

Figure I Mechanical ventilation induces pulmonary injury together with inflammation as well as YAP expression. Histological analysis (A) indicates that mechanical ventilation (MV) induces marked pulmonary injuries as reflected by increased histological scores (B) and wet/dry ratio (C). ELISA assays show that MV also induces excessive production of pro-inflammatory cytokines including TNF- $\alpha$ (D), IL-I $\beta$ (E), and IL-6 (F). In addition, MV induces YAP and its downstream gene CTGF expressions in pulmonary tissues $(\mathbf{G}$ and $\mathbf{H}) . * * * \mathrm{p}<0.001$ vs Control group. 


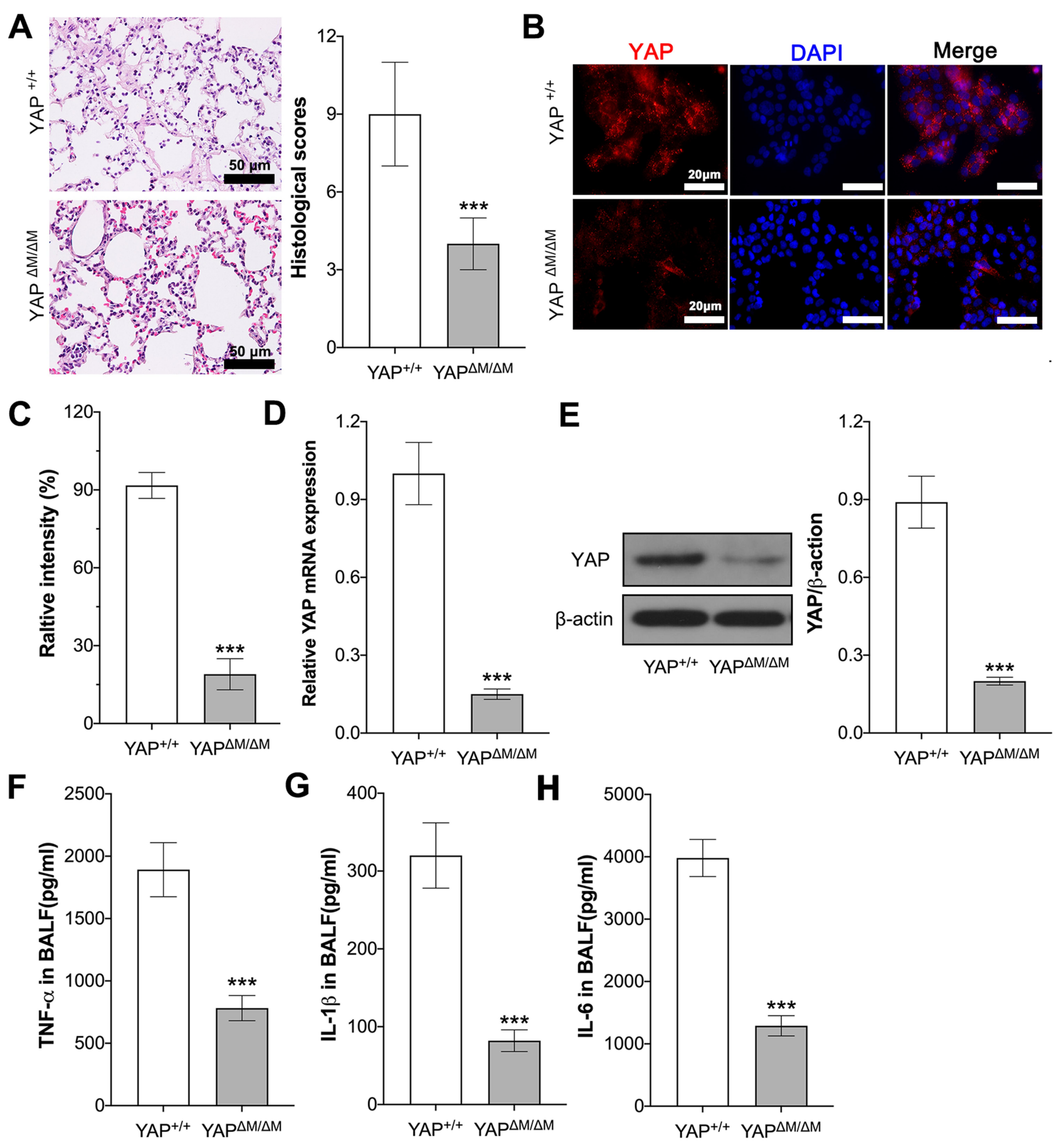

Figure 2 YAP deficiency protects mice against pulmonary injury following mechanical ventilation. Histological analysis (H\&E stain, 200x) (A) reveals that YAP deficiency attenuates pulmonary injury as reflected by decreased histological injury scores. Immunofluorescence staining (B and $\mathbf{C})$, RT-PCR (D) and western-blots (E) show marked decreases in YAP expression in isolated pulmonary macrophages. In addition, ELISA assays suggest that the expressions of pro-inflammatory cytokines including TNF- $\alpha(\mathbf{F})$, IL-I $\beta(\mathbf{G})$, and IL-6 $(\mathbf{H})$ are significantly downregulated in YAP-deficient macrophages as compared with controls. ${ }^{* * *} \mathrm{P}<0.00 \mathrm{I}$ vs $\mathrm{YAP}^{+/+}$group.

we first determined M2 polarization in injured lungs from mechanically ventilated mice. CD206 has been considered as a M2 macrophage marker, and an increase in the number of CD206 positive M2 macrophages was observed in $\mathrm{YAP}^{\Delta \mathrm{M} / \Delta \mathrm{M}}$ mice (Figure $3 \mathrm{~A}$ ). In addition, western-blots determined significant increases of M2 marker Arg1 protein expressions in $\mathrm{YAP}^{\Delta \mathrm{M} / \Delta \mathrm{M}}$ mice as compared with $\mathrm{YAP}^{+/+}$mice (Figure 3B). Next, pulmonary macrophages were isolated from $\mathrm{YAP}^{+/+}$and $\mathrm{YAP}^{\Delta \mathrm{M} /}$ $\triangle \mathrm{M}$ mice at the end of MV. Flow cytometric analysis revealed that the proportion of M2 macrophages of total macrophages was significantly higher in $\mathrm{YAP}^{\Delta \mathrm{M} /}$ 

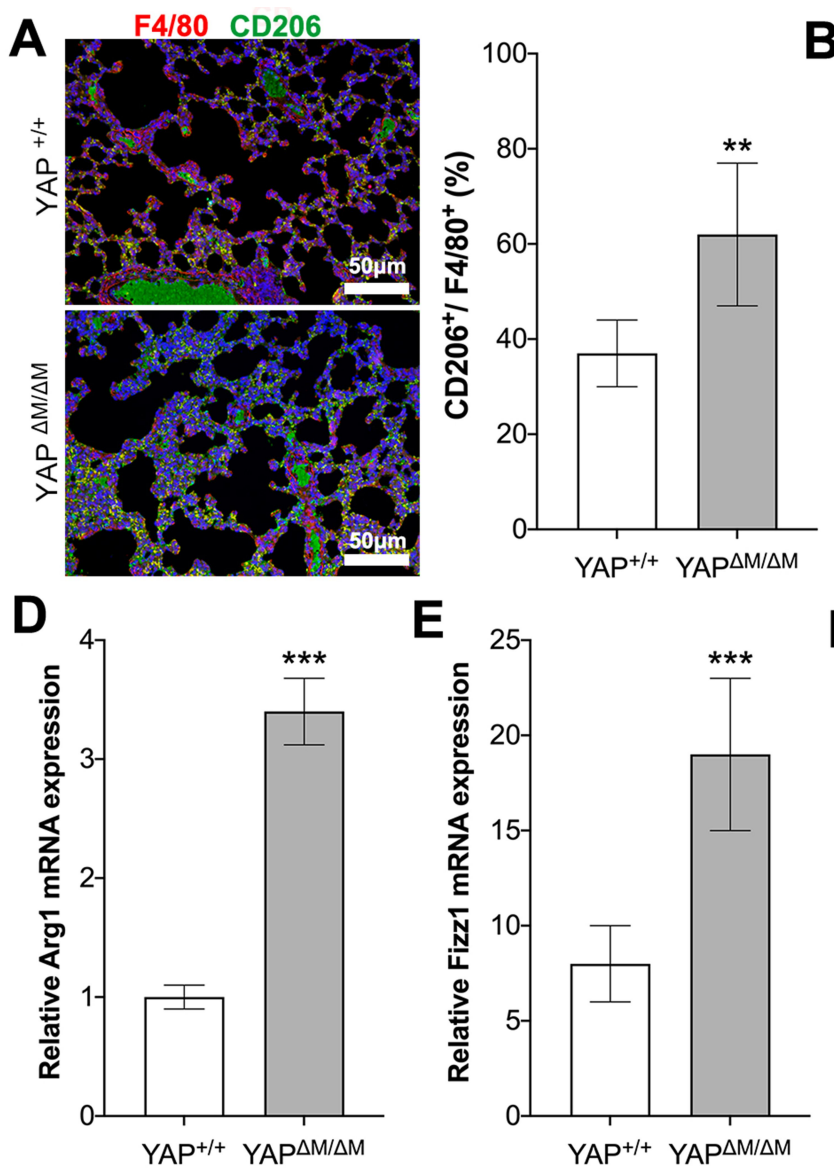

B

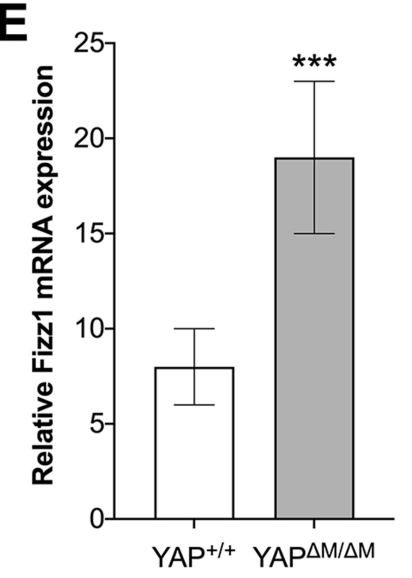

$\mathbf{F}$
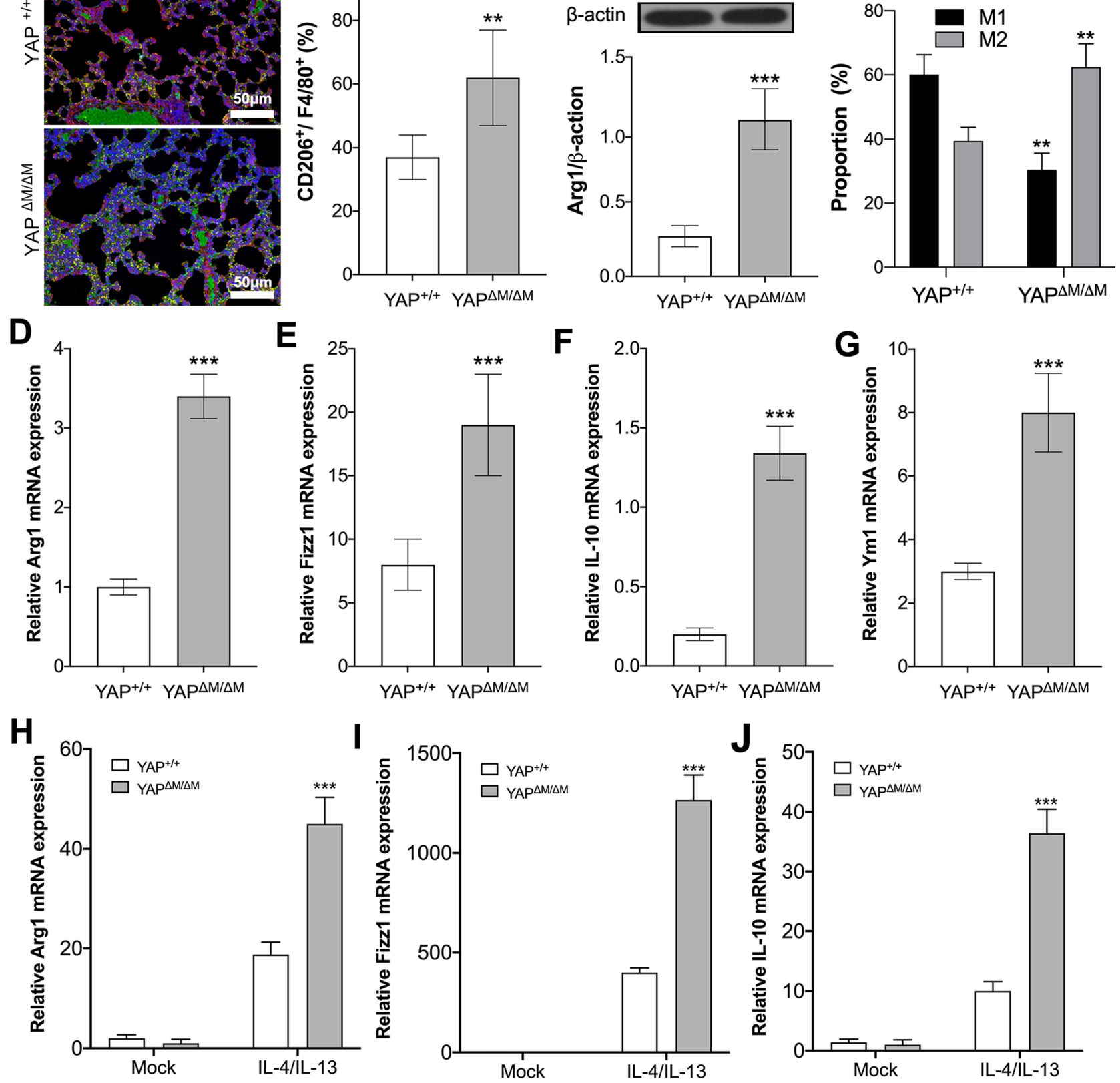

Figure 3 YAP deficiency enhances M2 macrophage polarization. (A) Immunofluorescence staining shows that the number of $C D 206^{+} / F 4 / 80^{+} M 2$ macrophages is increased in YAP-deficient mice. (B) The protein expression of M2 macrophage marker Argl is also upregulated in YAP-deficient mice. (C) The proportion of M2 macrophage of total macrophages was significantly higher in YAP-deficient mice than that of wild-type mice. (D-G) RT-PCR suggests that the expressions of M2 macrophage-related genes including Arg I, Fizz I, IL-I0 and Ym I are upregulated in YAP-deficient pulmonary macrophages. (H-J) Isolated bone-marrow-derived macrophages (BMM) were treated with IL-4/IL-I 3 for 24 hours. As compared to YAP ${ }^{+/+}$BMM, YAP-deficient BMM show significant increases in Arg- I, FizzI and IL-I0 mRNA expressions. **P < $0.0 \mathrm{I}$, *** $\mathrm{P}<0.00 \mathrm{I}$ vs $\mathrm{YAP}^{+/+}$or vector group.

$\Delta \mathrm{M}$ mice than that of $\mathrm{YAP}^{+/+}$mice. In contrast, the proportion of M1 macrophages was markedly decreased in $\mathrm{YAP}^{\Delta \mathrm{M} / \Delta \mathrm{M}}$ mice as compared with $\mathrm{YAP}^{+/+}$mice (Figure 3C). RT-PCR revealed that the mRNA expression of M2-related genes, including Arg1, Fizz, IL-10 and $\mathrm{Ym} 1$, were significantly enhanced in $\mathrm{YAP}^{\Delta \mathrm{M} / \Delta \mathrm{M}}$ mice as compared with $\mathrm{YAP}^{+/+}$mice (Figure 3D-G). To further confirm the function of YAP on M2 macrophage polarization, we used IL-4/IL-13 for the induction of M2 macrophages in vitro. Our results suggested that the expressions of M2 polarization markers in YAPdeficient macrophages (bone marrow-derived 
macrophages (BMM) from $\mathrm{YAP}^{\triangle \mathrm{M} / \Delta \mathrm{M}}$ mice) were significantly higher than that in wild-type macrophages $\left(\mathrm{BMM}\right.$ from $\mathrm{YAP}^{+/+}$mice) (Figure $3 \mathrm{H}-\mathrm{J}$ ).

\section{YAP Deficiency Inhibits MI Macrophage Polarization and Enhances Pro-Inflammatory Cytokine Production}

Next, we aim to determine the effect of YAP in M1 macrophage polarization. Immunofluorescence staining suggested that the number of pulmonary M1 macrophages was reduced in $\mathrm{YAP}^{\triangle \mathrm{M} / \Delta \mathrm{M}}$ mice (Figure $4 \mathrm{~A}$ ). The expression of iNOS, an M1 macrophage marker, was significantly decreased in $\mathrm{YAP}^{\Delta \mathrm{M} / \Delta \mathrm{M}}$ mice as compared with $\mathrm{YAP}^{+/+}$mice $(\mathrm{p}<0.001$, respectively) (Figure $4 \mathrm{~B}$ and C). In agreement of these findings, IL-6, IL-1 $\beta$ and TNF$\alpha$ mRNA expressions were markedly decreased in isolated macrophages from $\mathrm{YAP}^{\Delta \mathrm{M} / \Delta \mathrm{M}}$ mice as compared with that of $\mathrm{YAP}^{+/+}$mice $(p<0.001$, respectively) (Figure 4D-F). In addition, LPS and IFN- $\gamma$ were used to induce M1 macrophage polarization in vitro. Our results suggested that the expression of M1 macrophage markers including iNOS, IL-6 and TNF- $\alpha$ was significantly lower in YAP-deficient BMM than that of wild-type BMM cells $(p<0.001$, respectively) (Figure 4G-I).

\section{YAP Overexpression Inhibits M2 but Improves MI Macrophage Polarization}

In order to further determine the roles of YAP in the regulation of macrophage polarization, the induction of M1 and M2 polarization was performed using RAW264.7 cells after YAP plasmid transfection. The efficiency of transfection was determined by RT-PCR and western-blots (Figure 5A and B). YAP overexpression inhibits the induction of M2 macrophage-related genes (Arg-1, Fizz1 and IL-10) by IL-4/IL-13 stimulus in RAW264.7 cells (Figure 5C-E). However, YAP overexpression induced marked increases in the expression of M1 markers (iNOS, IL-6 and TNF- $\alpha$ ) in RAW264.7 cells ( $p<$ 0.001 , respectively) (Figure 5F-H). Together, these results suggested that YAP inhibits M2 macrophage polarization, while M1 macrophage polarization was enhanced by YAP overexpression.

\section{Discussion}

The major findings of this study can be summarized as follows: (1) mechanical ventilation for 12 hours induces marked pulmonary inflammation together with upregulated YAP expression; (2) YAP deficiency promotes M2 macrophage polarization while inhibits M1 polarization in vivo and in vitro. (3) YAP overexpression inhibits M2 polarization but improves M1 polarization; (4) YAP deficiency in macrophages protects mice against MVinduced pulmonary injury through the downregulation of inflammation by regulating M1/M2 polarization.

Although it has been well demonstrated that YAP acts as a transcriptional coactivator in tumor cells, ${ }^{20}$ its function in macrophages is limited. In fact, the expression level of YAP in immune cells including monocytes and macrophages is relatively low in mammals. ${ }^{11}$ However, increasing evidence indicate that YAP plays an important role in the regulation of M1/M2 macrophage polarization, ${ }^{11,21,22}$ which has been proposed to regulate pulmonary inflammation during ALI and ARDS. ${ }^{4,5}$ As a transcriptional coactivator, dephosphorylated YAP translocates into the nucleus where it interacts with transcription factors (TFs) or TFs possessing a PPXY motif ${ }^{23}$. Subsequently, YAP participates in the pathophysiological process through the induction of the transcription of target genes. ${ }^{24}$ These evidences indicated that YAP works at protein level in most manner. In the present study, we report for the first time that MV induces excessive expression of YAP at both mRNA and protein levels in pulmonary macrophages. Importantly, our study shows that YAP plays a dual role in the regulation of macrophage polarization in lungs during MV. In vitro experiments demonstrated that YAP drives macrophages towards M1 polarization while restricts M2 polarization. In addition, YAP deficiency down-regulated M1 polarization but improved M2 polarization in pulmonary tissues following 12 hours of mechanical ventilation. Together, those findings suggest that the induction of YAP in pulmonary macrophages probably contributes to the imbalance between M1/M2 polarization and subsequent inflammation after mechanical ventilation. Since pulmonary injury following mechanical ventilation is commonly associated with inflammation reaction, ${ }^{25}$ YAP could be a potential therapeutic target of mechanical ventilationinduced lung injury.

Our results suggested that the induction of YAP results in imbalance between M1/M2 macrophage polarization and excessive production of pro-inflammatory cytokines including Il-6, IL-1 $\beta$ and TNF- $\alpha$. However, the underlying mechanism by which YAP regulates M1/M2 macrophage polarization has not been documented in the present study. It has been demonstrated that the signal transducer and activator of transcription (STAT) proteins, interferon-regulatory 


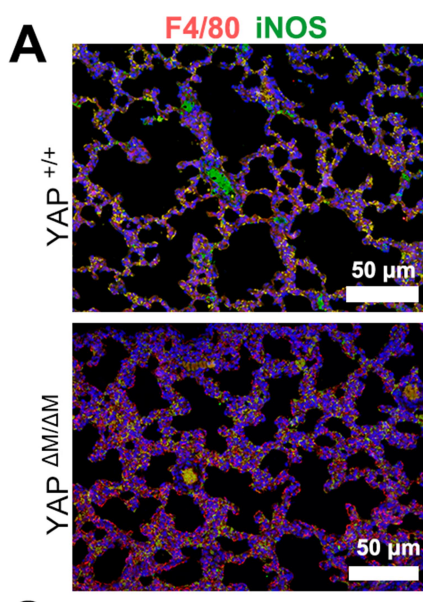

C

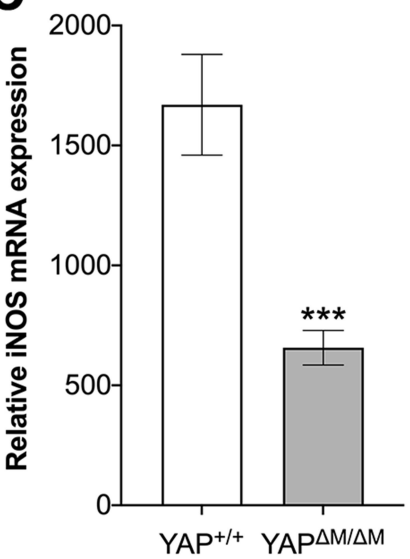

G

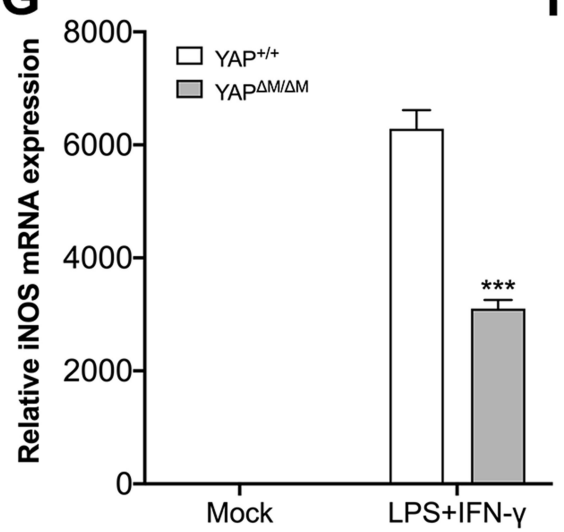

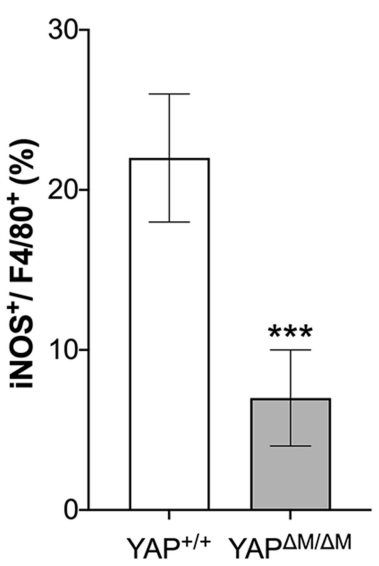

D

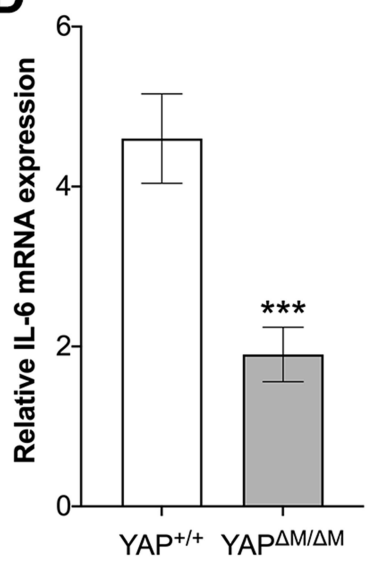

H
B

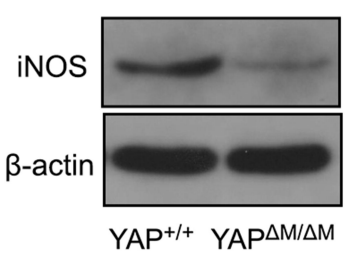

E

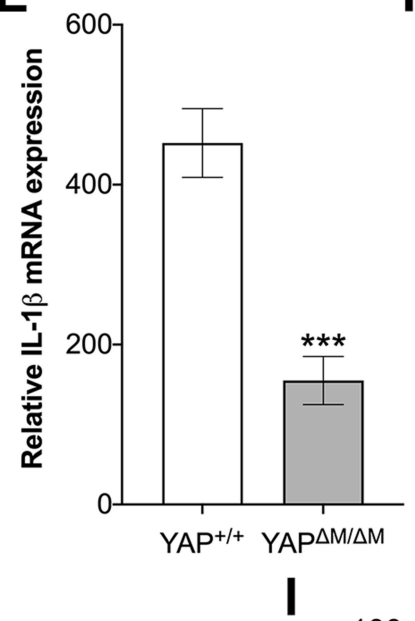

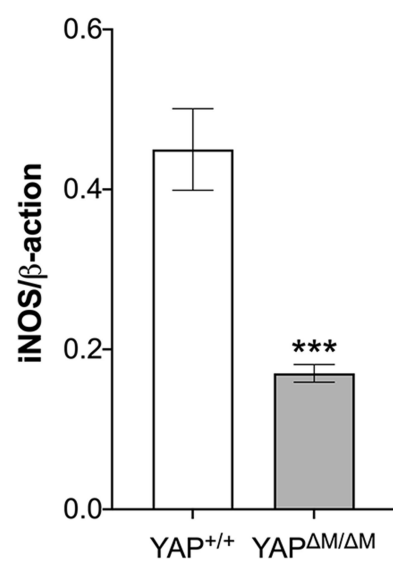

F

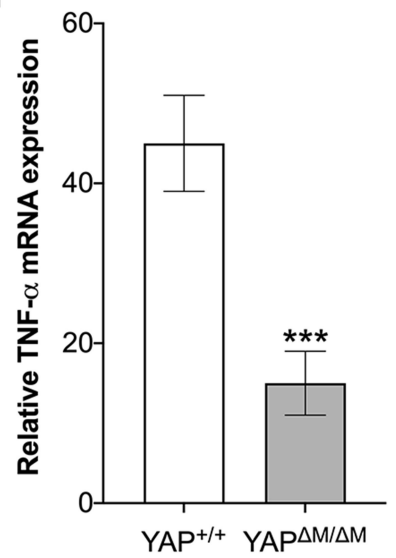

Figure 4 YAP deficiency inhibits MI macrophage polarization. (A) Immunofluorescence staining shows that the number of iNOS ${ }^{+} / \mathrm{F}^{2} / 80^{+} \mathrm{MI}$ macrophages is decreased in YAP-deficient mice. (B) The protein expression of MI macrophage marker iNOS is also downregulated in YAP-deficient mice. (C-F) RT-PCR suggests that the expressions of MI macrophage-related genes including iNOS, IL-6, IL-I $\beta$ and TNF- $\alpha$ are downregulated in YAP-deficient pulmonary macrophages. (G-I) Isolated bone-marrow-derived macrophages (BMM) were treated with LPS/IFN- $\gamma$ for 24 hours. As compared to YAP ${ }^{+/+}$BMM, YAP-deficient BMM show significant decreases in iNOS, IL-6, and TNF- $\alpha$ mRNA expressions. ${ }^{* * *} \mathrm{p}<0.00 \mathrm{I}$ vs $\mathrm{YAP}^{+/+}$or vector group.

factors (IRF) and CCAAT/enhancer-binding protein (C/EBP) are pivotal factors in M1/M2 macrophage polarization. ${ }^{26-28}$ However, whether the regulatory effects of YAP on M1/M2 macrophage polarization are associated with these factors is unclear. Previous studies have reported that YAP can bind to the promoter of IL- 6 and enhances IL- 6 production in bowel tissues. ${ }^{11}$ In addition, YAP participate in immune reprogramming in pancreatic ductal adenocarcinoma through the promotion of IL-6 expression. ${ }^{29}$ In the present study, we observed that YAP can promote IL-6 expression in RAW264.7 cells and YAP deficiency reduces IL-6 expression in pulmonary tissues after mechanical ventilation. Given the 

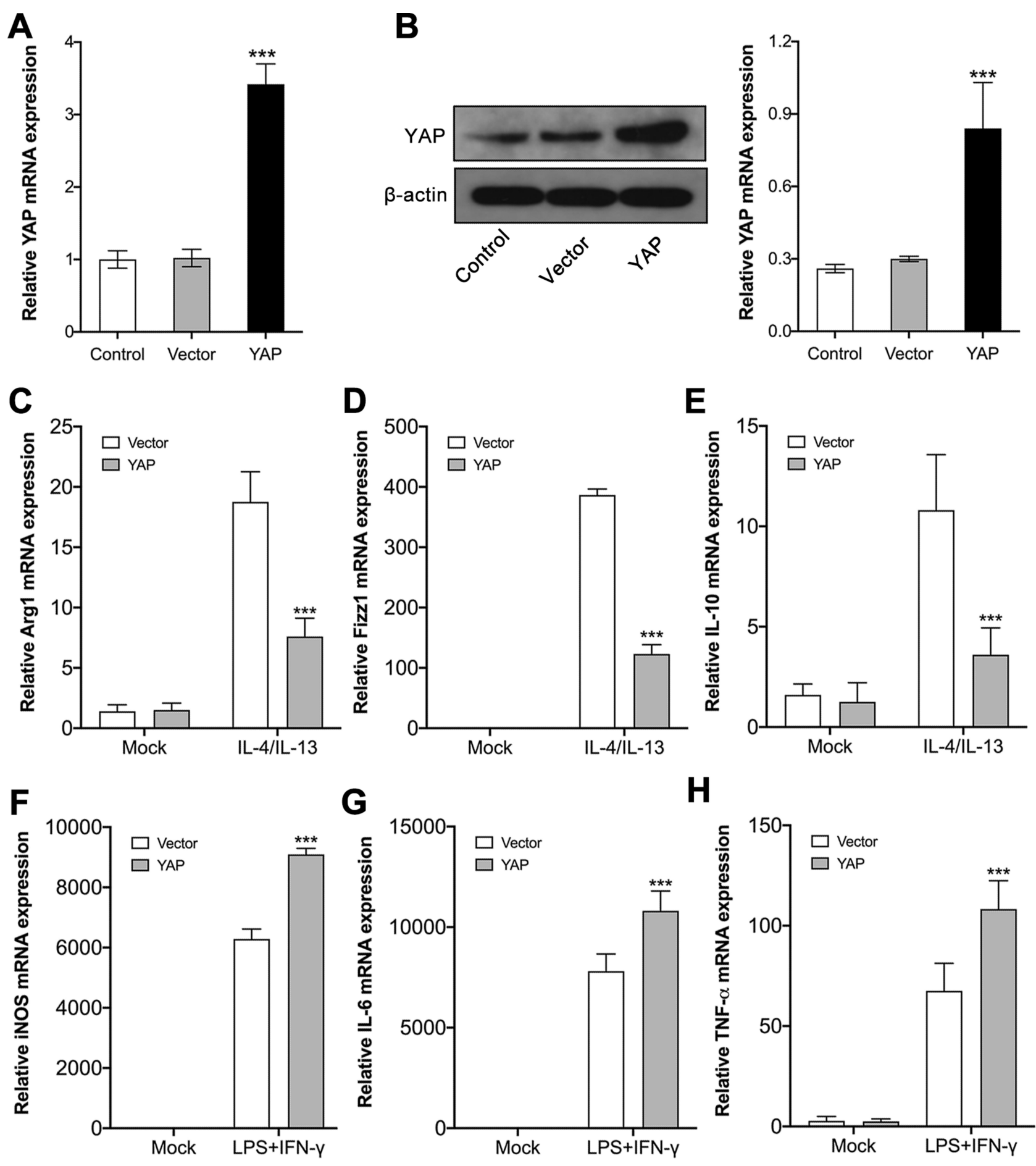

H

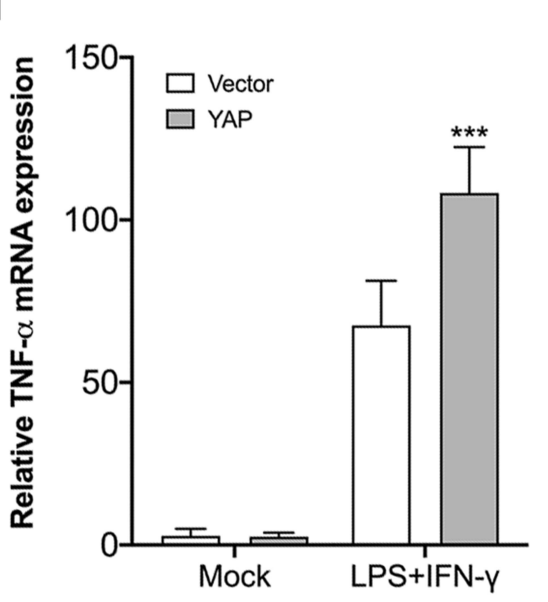

Figure 5 YAP overexpression inhibits M2 but enhances MI macrophage polarization. In vitro induction of MI and M2 polarization was performed using RAW264.7 cells after YAP plasmid or vector transfection. The transfection efficiency was determined by RT-PCR and western-blots (A and B). YAP overexpression significantly inhibited the expression of M2 macrophage-related genes (Arg-I, FizzI, IL-10) in RAW264.7 cells after IL-4/IL-I3 treatment (C-E). YAP overexpression markedly improves the expression of MI macrophage-related genes (iNOS, IL-6, TNF- $\alpha$ ) in RAW264.7 cells after IL-4/IL-I3 treatment $(\mathbf{F}-\mathbf{H})$. ${ }^{* * *} \mathrm{p}<0.00 \mathrm{I}$ vs YAP ${ }^{+/+}$or vector group.

rapidly accumulating evidence supporting the IL-6 targeted therapy in inflammatory autoimmune diseases, ${ }^{30}$ our results suggest that targeting YAP in macrophages should be a possible therapy for mechanical ventilation-induced pulmonary inflammation and subsequent injury. On the other side, the induction of YAP inhibits M2 macrophage marker Arg1 expression, which was in line with previous findings in hepatocytes. ${ }^{31}$ Moreover, YAP inhibits the 
production of anti-inflammatory cytokines in vivo and in vitro. Importantly, as a key metabolic enzyme, Arg1 is associated with a wide range of inflammatory diseases. ${ }^{32}$ Again, our results suggest an important role of YAP in the process of inflammation.

In conclusion, the early induction of YAP in macrophages probably contributes to pulmonary inflammation following mechanical ventilation. Therefore, YAP could be considered as a potential therapeutic target for VILI. However, further studies are required to elucidate the underlying mechanism by which YAP regulates M1/M2 macrophage polarization.

\section{Funding}

There is no funding to report.

\section{Disclosure}

The authors declare no conflicts of interest for this work.

\section{References}

1. Tobin M, Manthous C. Mechanical ventilation. Am J Respir Crit Care Med. 2017;196:P3-P4.

2. Esteban A, Frutos-Vivar F, Muriel A, et al. Evolution of mortality over time in patients receiving mechanical ventilation. Am J Respir Crit Care Med. 2013;188:220-230. doi:10.1164/rccm.201212-2169OC

3. Choi YB, Lee J, Park J, Jun YH. Impact of prolonged mechanical ventilation in very low birth weight infants: results from a national cohort study. J Pediatr. 2018;194:34-39.e3. doi:10.1016/j.jpeds. 2017.10.042

4. Tang L, Zhang H, Wang C, Li H, Zhang Q, Bai J. M2A and M2C macrophage subsets ameliorate inflammation and fibroproliferation in acute lung injury through interleukin 10 pathway. Shock. 2017;48:119-129. doi:10.1097/SHK.0000000000000820

5. Garnier M, Gibelin A, Mailleux AA, et al. Macrophage polarization favors epithelial repair during acute respiratory distress syndrome. Crit Care Med. 2018;46:e692-e701. doi:10.1097/CCM.0000 000000003150

6. Murray PJ, Allen JE, Biswas SK, et al. Macrophage activation and polarization: nomenclature and experimental guidelines. Immunity. 2014;41:14-20. doi:10.1016/j.immuni.2014.06.008

7. Atri C, Guerfali FZ, Laouini D. Role of human macrophage polarization in inflammation during infectious diseases. Int $\mathrm{J} \mathrm{Mol} \mathrm{Sci}$ 2018;19:1801. doi:10.3390/ijms19061801

8. Ohradanova-Repic A, Machacek C, Charvet C, et al. Extracellular purine metabolism is the switchboard of immunosuppressive macrophages and a novel target to treat diseases with macrophage imbalances. Front Immunol. 2018;9:852. doi:10.3389/fimmu.2018.00852

9. Hsu PC, Yang CT, Jablons DM, You L. The role of yes-associated protein (YAP) in regulating programmed death-ligand 1 (PD-L1) in thoracic cancer. Biomedicines. 2018;6:114. doi:10.3390/ biomedicines6040114

10. Lv Y, Kim K, Sheng Y, et al. YAP controls endothelial activation and vascular inflammation through TRAF6. Circ Res. 2018;123:43-56. doi:10.1161/CIRCRESAHA.118.313143

11. Zhou X, Li W, Wang S, et al. YAP aggravates inflammatory bowel disease by regulating M1/M2 macrophage polarization and gut microbial homeostasis. Cell Rep. 2019;27:1176-1189.e5. doi:10.1016/j.celrep.2019.03.028
12. Tu M, Yao Y, Qiao FH, Wang L. The pathogenic role of connective tissue growth factor in osteoarthritis. Biosci Rep. 2019;39: BSR20191374. doi:10.1042/BSR20191374

13. Venosa A, Malaviya R, Choi H, Gow AJ, Laskin JD, Laskin DL. Characterization of distinct macrophage subpopulations during nitrogen mustard-induced lung injury and fibrosis. Am J Respir Cell Mol Biol. 2016;54:436-446. doi:10.1165/rcmb.2015-01200C

14. Xie Y, Wang Y, Liu K, Li X. Correlation analysis between mechanical power, transforming growth factor- $\beta 1$, and connective tissue growth factor levels in acute respiratory distress syndrome patients and their clinical significance in pulmonary structural remodeling. Medicine (Baltimore). 2019;98(29):e16531. doi:10.1097/MD.00000 00000016531

15. Couto M, Cates C. Laboratory guidelines for animal care. Methods Mol Biol. 2019;1920:407-430.

16. Arora S, Olszewski MA, Tsang TM, McDonald RA, Toews GB, Huffnagle GB. Effect of cytokine interplay on macrophage polarization during chronic pulmonary infection with Cryptococcus neoformans. Infect Immun. 2011;79:1915-1926. doi:10.1128/ IAI.01270-10

17. Herb M, Farid A, Gluschko A, Krönke M, Schramm M. Highly efficient transfection of primary macrophages with in vitro transcribed mRNA. J Vis Exp. 2019;153:e60143.

18. Zhu R, Lei YQ, Zhao DC. Overexpression of CXCL14 alleviates ventilator-induced lung injury through the downregulation of PKM2-mediated cytokine production. Mediators Inflamm. 2020;2020:7650978. doi:10.1155/2020/7650978

19. Luo Y, Li N, Chen H, et al. Spatial and temporal changes in extracellular elastin and laminin distribution during lung alveolar development. Sci Rep. 2018;8:8334. doi:10.1038/s41598-01826673-1

20. Wang G, Lu X, Dey P, et al. Targeting YAP-dependent MDSC infiltration impairs tumor progression. Cancer Discov. 2016;6:80-95. doi:10.1158/2159-8290.CD-15-0224

21. Feng Y, Liang Y, Zhu X, et al. The signaling protein Wnt5a promotes TGF $\beta 1$-mediated macrophage polarization and kidney fibrosis by inducing the transcriptional regulators Yap/Taz. J Biol Chem. 2018;293:19290-19302. doi:10.1074/jbc.RA118.005457

22. Huang YJ, Yang CK, Wei PL, et al. Ovatodiolide suppresses colon tumorigenesis and prevents polarization of M2 tumor-associated macrophages through YAP oncogenic pathways. $J$ Hematol Oncol. 2017;10:60. doi:10.1186/s13045-017-0421-3

23. Piccolo S, Dupont S, Cordenonsi M. The biology of YAP/TAZ: hippo signaling and beyond. Physiol Rev. 2014;94:1287-1312. doi:10.1152/ physrev.00005.2014

24. Dupont S. Role of YAP/TAZ in cell-matrix adhesion-mediated signalling and mechanotransduction. Exp Cell Res. 2016;343:42-53. doi:10.1016/j.yexcr.2015.10.034

25. Qiu JL, Song BL, Wang YJ, Zhang FT, Wang YL. Role of glutamine in the mediation of E-cadherin, p120-catenin and inflammation in ventilator-induced lung injury. Chin Med J (Engl). 2018; 131:804-812. doi:10.4103/0366-6999.228230

26. Gao S, Mao F, Zhang B, et al. Mouse bone marrow-derived mesenchymal stem cells induce macrophage M2 polarization through the nuclear factor- $\mathrm{\kappa B}$ and signal transducer and activator of transcription 3 pathways. Exp Biol Med (Maywood). 2014;239:366-375. doi:10. $1177 / 1535370213518169$

27. Chistiakov DA, Myasoedova VA, Revin VV, Orekhov AN, Bobryshev YV. The impact of interferon-regulatory factors to macrophage differentiation and polarization into M1 and M2. Immunobiology. 2018;223:101-111. doi:10.1016/j.imbio.2017.10.005

28. Ponomarev ED, Veremeyko T, Barteneva N, Krichevsky AM, Weiner HL. MicroRNA-124 promotes microglia quiescence and suppresses EAE by deactivating macrophages via the C/EBP- $\alpha$-PU.1 pathway. Nat Med. 2011;17:64-70. doi:10.1038/nm.2266 
29. Murakami S, Shahbazian D, Surana R, et al. Yes-associated protein mediates immune reprogramming in pancreatic ductal adenocarcinoma. Oncogene. 2017;36:1232-1244. doi:10.1038/onc.2016.288

30. Yao X, Huang J, Zhong H, et al. Targeting interleukin-6 in inflammatory autoimmune diseases and cancers. Pharmacol Ther. 2014;141:125-139. doi:10.1016/j.pharmthera.2013.09.004
31. Yimlamai D, Christodoulou C, Galli GG, et al. Hippo pathway activity influences liver cell fate. Cell. 2014;157:1324-1338. doi:10.1016/j.cell.2014.03.060

32. Zhu M, Goetsch SC, Wang Z, et al. FoxO4 promotes early inflammatory response upon myocardial infarction via endothelial Arg1. Circ Res. 2015;117:967-977. doi:10.1161/CIRCRESAHA.115.306919

\section{Publish your work in this journal}

The Journal of Inflammation Research is an international, peerreviewed open-access journal that welcomes laboratory and clinical findings on the molecular basis, cell biology and pharmacology of inflammation including original research, reviews, symposium reports, hypothesis formation and commentaries on: acute/chronic inflammation; mediators of inflammation; cellular processes; molecular mechanisms; pharmacology and novel anti-inflammatory drugs; clinical conditions involving inflammation. The manuscript management system is completely online and includes a very quick and fair peerreview system. Visit http://www.dovepress.com/testimonials.php to read real quotes from published authors. 